\title{
Spatial and Temporal Expression Patterns of Two Sodium Channel Genes in Drosophila
}

\author{
Chang-Sook Hong and Barry Ganetzky \\ Laboratory of Genetics, University of Wisconsin, Madison, Wisconsin 53706
}

\begin{abstract}
Genetic and molecular studies have identified two different sodium channel genes in Drosophila, para and DSC1. The functional contributions of the para-encoded channel have been inferred from analysis of mutant phenotypes. However, no mutations of DSC1 have been identified, so the in vivo functions of the channel it encodes are not yet known. To learn more about the possible functions of DSC 1 in the Drosophila nervous system compared with those of para, we have characterized the expression patterns of these two sodium channel genes at embryonic, larval, pupal, and adult stages by tissue in situ hybridization. para encodes the predominant type of sodium channel and is ubiquitously transcribed throughout the CNS and PNS at all developmental stages. The expression pattern of DSC1 is very different from para during embryonic and larval stages during which there are very few DSC1-expressing cells in either the CNS or PNS. Double-labeling studies suggest that some of these cells are non-neuronal. However, in pupal and adult stages, para and $D S C 1$ have completely overlapping patterns of expression in the CNS and retina. In the pupal and adult, PNS expression of these genes is still distinct because only para transcripts are detected in wing sensory neurons. The strong and widespread expression of DSC1 in the CNS of pupae and adults suggests that the $D S C 1$ channels are likely to provide an important function in neurons during these stages. Since most, if not all, neurons in the pupal and adult CNS express both para and $D S C 1$, these two sodium channel genes probably subserve distinct functions within these cells. Our results provide the background for elucidating the respective in vivo contributions of para and DSC1 to neuronal excitability and for dissecting the regulatory mechanisms that underlie their different patterns of expression.
\end{abstract}

[Key words: Drosophila, para, DSC1, sodium channel, gene expression, in situ hybridization]

The electrical properties of a neuron are largely shaped by the types and relative abundance of ion channels that are expressed. Generally, ion channel genes are members of families in which two or more genes encode channels with similar, but not iden-

\footnotetext{
Received Dec. 20, 1993; revised Mar. 16, 1994; accepted Mar. 24, 1994.

We thank Jon Karpilow, Janna McLean, Richard Ordway, and Justin Thackeray for comments on the manuscript and members of Mike Hoffmann's laboratory for advice and help with in situ hybridization and histological techniques. This work was supported by National Institutes of I Iealth Grant GM43100. This is paper 3393 from the Laboratory of Genetics, University of Wisconsin, Madison.

Correspondence should be addressed to Dr. Barry Ganetzky, Laboratory of Genetics, 445 Henry Mall, University of Wisconsin, Madison, WI 53706.

Copyright (C) 1994 Society for Neuroscience $0270-6474 / 94 / 145160-10 \$ 05.00 / 0$
}

tical, functional properties (Hille, 1992). The signaling capabilities of a given neuron may vary, depending on which member of an ion channel family is expressed. For example, in mammals, at least five genes encode sodium channel polypeptides, three of which are expressed in neurons and two in skeletal muscle (Noda et al., 1986; Kayano et al., 1988; Rogart et al., 1989; Trimmer et al., 1989; Kallen et al., 1990). Each of these genes has a distinctive temporal and spatial pattern of expression, resulting in distributions of sodium channel types that are largely nonoverlapping (reviewed by Mandel, 1992). Thus, in rat brain the mRNA levels of neuronal sodium channel types II and III increase during neurogenesis from embryonic day 10 to birth. Type II mRNA levels remain high in adults, whereas type III decreases to a lower level. Type I mRNA does not show appreciable abundance until birth. The type II and type III genes are expressed throughout the CNS, but only sparsely in the PNS. Expression of the type I gene is most abundant in caudal regions of the brain and in the spinal cord. The implication from the existence of this family of sodium channel genes, each with its own pattern of expression, is that the different sodium channel polypeptides have distinctive functional properties that are important in determining the characteristic firing properties of a particular type of neuron. However, it has not yet been possible experimentally to determine the respective functional contributions of each sodium channel type to the electrophysiological properties of the neurons in which they are expressed.

Because of the numerous genetic and molecular tools available, Drosophila is well suited for investigating problems of this type. At least two different sodium channel structural genes have been identified in Drosophila. The para (paralytic) locus was first identified on the basis of mutations causing a temperaturesensitive paralytic phenotype (Suzuki et al., 1971; Siddiqi and Benzer, 1976). Electrophysiological experiments demonstrated that paralysis was correlated with a temperature-dependent block in the propagation of neuronal action potentials (Siddiqi and Benzer, 1976; Wu and Ganetzky, 1980). Subsequently, molecular analysis of this gene revealed that it encoded a sodium channel polypeptide sharing about $60 \%$ amino acid identity with rat brain sodium channels (Loughney et al., 1989; Ramaswami and Tanouye, 1989). Another presumptive sodium channel gene, DSCl (Drosophila sodium channel), was cloned on the basis of its homology to vertebrate sodium channel probes (Salkoff et al., 1987; Ramaswami and Tanouye, 1989). The importance of the $D S C I$ channel to neuronal excitability is not known because mutations affecting its function have not yet been isolated. However, the extensive sequence divergence between para and $D S C 1$ suggests that the encoded polypeptides may have distinct physiological functions in the Drosophila nervous system. However, 
only limited characterization of the expression of these genes in pupae and adults has been reported (Tseng-Crank et al., 1991; Amichot et al., 1993), so it is not yet known whether the two Drosophila sodium channel types are differentially distributed in the nervous system throughout development. To study the expression of these genes in detail, we have used tissue in situ hybridization to characterize their spatial and temporal pattern of transcription from embryonic through adult stages. Our results demonstrate that para is ubiquitously expressed throughout the CNS and PNS at all developmental stages. In contrast, $D S C 1$ has a restricted pattern of expression in embryos that shows little, if any, overlap with para. Surprisingly, however, there is almost complete overlap in the expression of para and $D S C l$ in the CNS of pupae and adults. These results provide the background for assessing the respective in vivo contributions of para and $D S C 1$ to neuronal excitability and for elucidating the regulatory mechanisms that underlie their different patterns of expression.

\section{Materials and Methods}

Fly stocks. Drosophila melanogaster were raised on standard Drosophila medium at $25^{\circ} \mathrm{C}$. The wild-type strain used in all experiments was Canton Special (CS). The null mutation paralkz has been previously described (Loughney et al., 1989) and was used as a negative control for the specificity of the para in situ probes.

Hybridization probes. A mixed probe synthesized from three DNA templates was used for detection of para transcripts: the $0.29 \mathrm{~kb}$ EcoRIBamHI fragment of cDNA ZS10.3, the $0.69 \mathrm{~kb}$ BglI-ClaI fragment of cDNA ZS10.3, and the $1.0 \mathrm{~kb}$ EcoRI-MspI fragment of cDNA GS12.1 (Loughney et al., 1989). These cDNA fragments encoded the cytoplasmic domains of the amino terminus and the linking regions between the first and second or second and third homology domains of the para polypeptide. These regions show no conservation between the para polypeptide and other known sodium channel polypeptides. Except for the 24 nucleotide segment corresponding to alternative exon b present in the $0.69 \mathrm{~kb} \mathrm{BglI}-\mathrm{ClaI}$ fragment, all templates contained exon sequences common to all para transcripts (Loughney et al., 1989). Templates for synthesis of $D S C I$ probes were either the $1.7 \mathrm{~kb}$ cDNA B1, which encodes the region distal to the third homology domain of the DSCl polypeptide (Ramaswami and Tanouye, 1989), or a PCR-amplified fragment that corresponds to the first cytoplasmic linking region. Identical results were obtained with either probe. Digoxigenin-labeled DNA probes were synthesized by random priming according to manufacturer's instructions (Genius kit, Boehringer-Mannheim). In some experiments, increased sensitivity was obtained by using digoxigeninlabeled single-stranded RNA probes. RNA probes were made by in vitro transcription according to manufacturer's instructions (BoehringerMannheim).

In situ hybridization. Nonradioactive in situ localization of RNA in whole embryos was done as described by Tautz and Pfeifle (1989) and Masucci et al. (1990) with some modifications. Embryos (0-16 hr) were prefixed by shaking for 20-30 min in heptane saturated with $10 \%$ formaldehyde, PBS (PBS $=10 \mathrm{~mm} \mathrm{KPO}, 140 \mathrm{~mm} \mathrm{NaCl}$, pH 7.2), $50 \mathrm{~mm}$ EGTA. To devitellinize the embryos the aqueous phase and most of the heptane were removed, methanol was added, and the embryos were shaken vigorously for several minutes. The embryos were then rinsed with methanol and then with $100 \%$ ethanol several times and stored at $-20^{\circ} \mathrm{C}$. Embryos were rehydrated by rinsing once in $50 \%$ ethanol, $50 \%$ PBT (PBT $=$ PBS $+0.1 \%$ Tween) and then three times in PBT. Following rehydration, embryos were fixed for $15 \mathrm{~min}$ on ice in $5 \%$ formaldehyde, PBS, and then for $15 \mathrm{~min}$ at room temperature in $5 \%$ formaldehyde, PBT. Fixed embryos were treated with proteinase $K$, washed, prehybridized, hybridized, and washed as described in Tautz and Pfeifle (1989). Hybridization was done for $24-36 \mathrm{hr}$ at $48^{\circ} \mathrm{C}$ for DNA probes and at $55^{\circ} \mathrm{C}$ for RNA probes. Embryos were incubated with preabsorbed alkaline phosphatase-conjugated antidigoxygenin antibody, diluted 1:2000, for $1 \mathrm{hr}$ at room temperature. After color development, embryos were mounted in glycerol or dehydrated in ethanol and then mounted in methyl salicylate.

Larval brain and imaginal discs were dissected out in PBS before fixing in 5\% formaldehyde, PBS. Pupal heads and wings from $25 \mathrm{hr}$ pupae were dissected out from the pupal case and cuticular membrane and then fixed in 5\% formaldehyde, PBS. Dissected tissues were hybridized in situ by the same procedures as described above for embryos. After the color reaction, larval ganglia and pupal heads were dehydrated in ethanol and cleared and mounted in methyl salicylate and then embedded in plastic resin (Ernest Fullam and Co.) as described by Reuter and Scott (1990). After overnight polymerization, the tissues were sectioned at $4 \mu \mathrm{m}$ thickness with an ultracut microtome using glass knives.

Heads from $72 \mathrm{hr}$ pupae and adults were frozen in Tissuetek O.C.T. compound (Lab-Tek Division, Miles) on a block of dry ice and then sectioned at $10 \mu \mathrm{m}$ thickness using a cryostat. Most procedures following sectioning were modified from a protocol designed for radioactive probes (Hafen and Levine, 1986). Tissue sections were fixed, treated with pronase, and prehybridized as described by Hafen and Levine (1986). Hybridization with digoxigenin-labeled RNA or DNA probes was carried out for $18-24 \mathrm{hr}$ at $45^{\circ} \mathrm{C}$ in a moist chamber. After hybridization, the slides were washed five times for $10 \mathrm{~min}$ each in $2 \times \mathrm{SSC}(\mathrm{SSC}=0.15$ $\mathrm{M} \mathrm{NaCl}, 0.015 \mathrm{M} \mathrm{Na}$-citrate), twice in $1 \times \mathrm{SSC}$, and twice in $0.5 \times \mathrm{SSC}$ at $45^{\circ} \mathrm{C}$. Color development was as described for whole embryos. After staining, the slides were dehydrated through an ethanol/xylene series and mounted with Permount.

Double labeling with anti-HRP immunohistochemistry. After in situ hybridization as described above, embryos were incubated for $1 \mathrm{hr}$ with anti-HRP antibody (Sigma; diluted 1:200), washed, and incubated for $1 \mathrm{hr}$ with horseradish peroxidase-conjugated goat anti-rabbit IgG (Sigma; diluted 1:500). Detection was done according to manufacturer's recommendations. After color development, embryos were mounted in $90 \%$ glycerol and the ventral nerve cord and the PNS were dissected out for flat mounting.

\section{Results}

Embryonic expression patterns of para and $\mathrm{DSCl}$ are different During Drosophila neurogenesis, neuroblasts begin to segregate from the ectoderm at the beginning of stage 9 and undergo asymmetric division to generate ganglion mother cells. In turn, these cells divide symmetrically to produce pairs of postmitotic neurons through stage 12 (Campos-Ortega and Hartenstein, 1985; Campos-Ortega and Jan, 1991). We used a para cDNA restriction fragment as a probe for tissue in situ hybridization to examine expression of para transcripts in the developing nervous system. In whole-mount embryos para expression is not observed prior to stage 12 but becomes detectable after early stage 13 (Fig. $1 A, C$ ). By using genomic DNA probes corresponding to the $5^{\prime}$ untranslated leader, para transcripts could be detected at late stage 12 , about 50 min earlier (data not shown) than with probes derived from the translated region. Thus, transcription of para probably initiates during late stage 12 in neurons but not in neuroblasts.

At stage 13, para transcripts are detected in the ventral nerve cord and in the antennomaxillary complex. Besides the neurons in the antennomaxillary complex, only a few other cells in the peripheral nervous system (PNS) show any para expression at this time. Because sensory neurons develop sequentially in Drosophila and only a few are present by stage 13 (Hartenstein, 1988), the stained cells in the periphery probably include the first sensory neurons to appear. Development of the PNS is complete by stage 15 , at which time most, and perhaps all, sensory neurons express para (Fig. $1 E$ ). In the CNS, the para transcript is detected continuously and strongly from stage 13 onward (Fig. 1C,G). Antibodies to horseradish peroxidase (HRP) recognize most neuronal cell bodies and axon tracts in the Drosophila nervous system (Jan and Jan, 1982). Double labeling experiments using anti-HRP antibodies in addition to probes for the para transcript were carried out to confirm the neuronal identity of para-expressing cells and to determine the location of these cells relative to axon tracts. As shown in Figure 2, $A$ and $B$, there are clusters of para-expressing neurons near the 


\section{para}
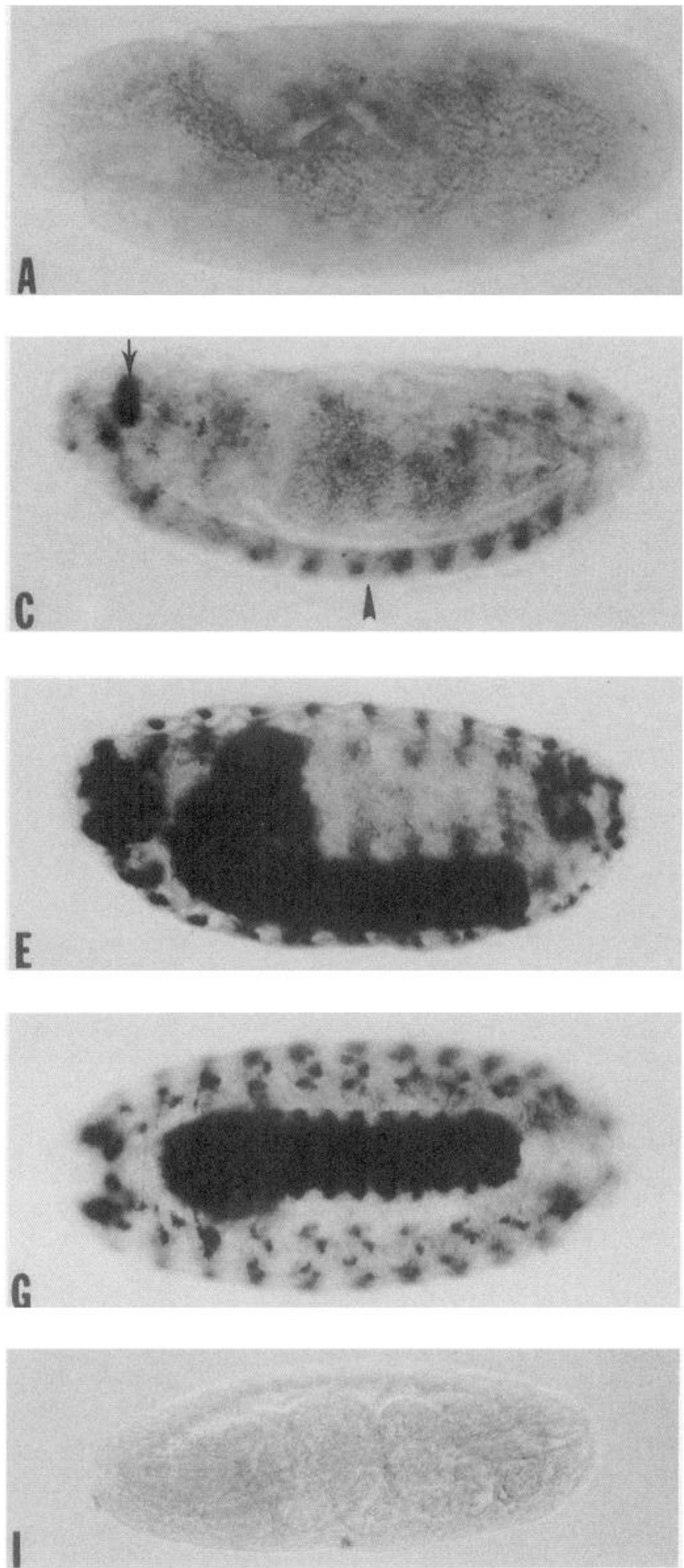
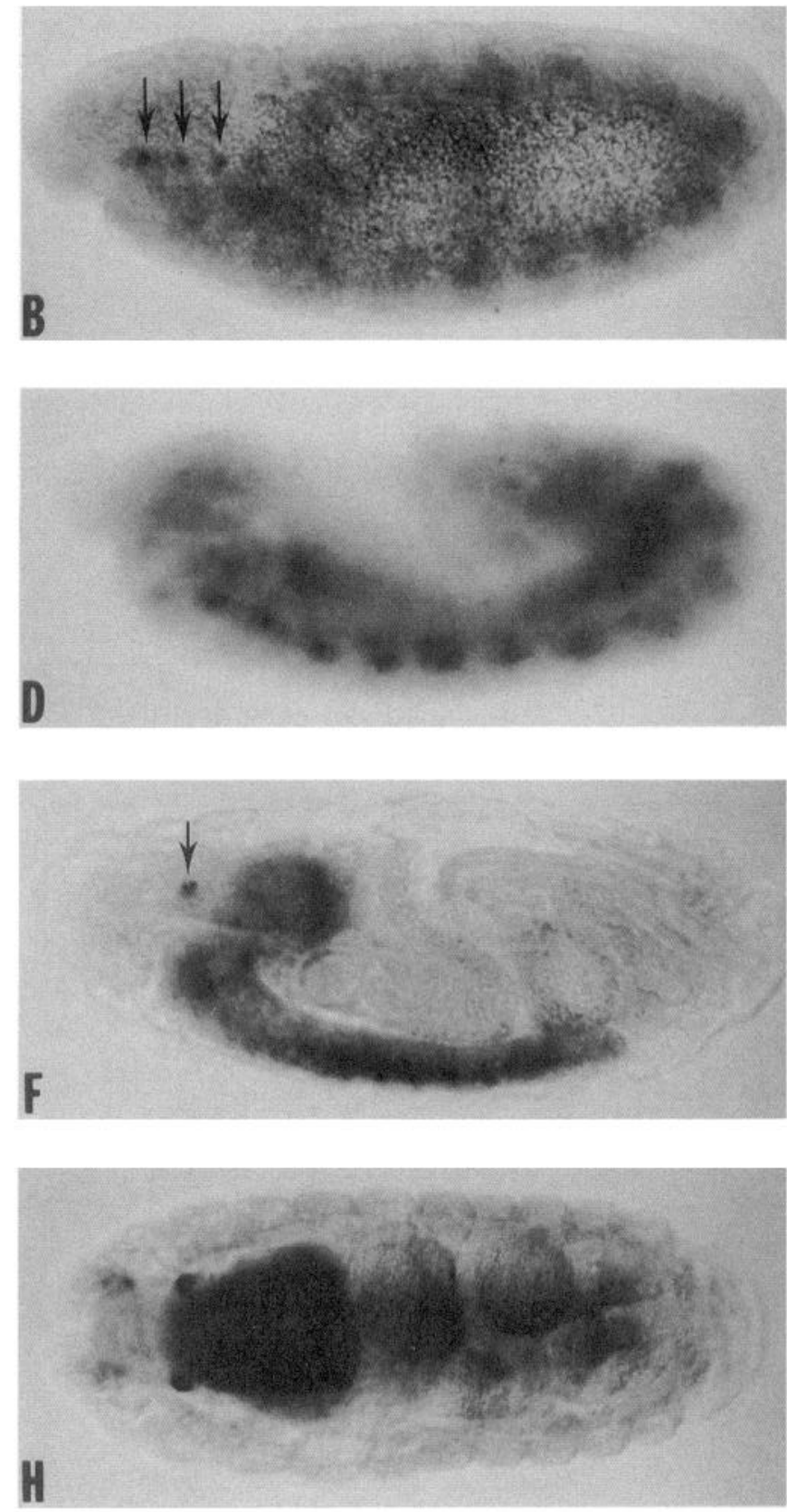

Figure 1. Analysis of embryonic expression of para and DSC1 sodium channel genes by in situ hybridization. Left panels (A, $C, E$, $G$, and $I)$ show para expression, and right panels $(B, D, F$, and $H), D S C 1$ expression. $A-F$ are lateral views of wild-type embryos of various stages. The embryos are oriented with anterior to the left and ventral down. $A$, Stage 12 embryo hybridized with para probe. No detectable staining is seen. $B$, Stage 12 embryo hybridized with $D S C 1$ probe. Expression can be observed in some cells in the developing stomatogastric ganglion (arrows) and germ band. $C$, Stage 13 embryo hybridized with para probe. Staining is present in the antennomaxillary complex (arrow) and ventral nerve cord (arrowhead). D, Late stage 12 embryo hybridized with $D S C 1$ probe. Staining appears in segmentally repeating cells along the ventral cord. E, Stage 
midline and at the lateral margins of the CNS close to the posterior commissures in every segment. In addition, double labeling experiments in the PNS indicate that the group of neurons expressing the HRP antigen is indistinguishable from the para-expressing cells (Fig. 2E,F,H). Thus, by stage 17, the final stage of embryonic development, para appears to be expressed in most, if not all, neurons in both the CNS and PNS.

To confirm the specificity of the probes for the para transcript, in situ hybridization was carried out on embryos produced by FM7/para ${ }^{l k 2}$ mothers. The para ${ }^{1 / 2}$ allele is a null mutation associated with an inversion breakpoint within the open reading frame of para (Loughney et al., 1989). The majority of parak homozygous embryos have been found to survive through embryogenesis until hatching (unpublished observations). When $F M 7 /$ para $^{l / 2}$ females are crossed to $F M 7 / Y$ males, about $25 \%$ of the resulting embryos should be para ${ }^{l k 2 / Y}$ mutant males and fail to express the para transcript. Examination of 141 late stage embryos from this cross revealed 34 (24\%) that did not hybridize with the para probes (Fig. 1I), demonstrating the specificity of the probes for the para transcript.

$D S C 1$ is first detected somewhat earlier than para at early stage 12 prior to germ band retraction (Fig. $1 B$ ). At this stage, several $D S C 1$-expressing cells appear in the dorsal wall of the foregut. The position of these cells appears to correspond to that of the stomatogastric nervous system, which gives rise to the frontal and parapharyngeal ganglia during stage 15 (CamposOrtega and Hartenstein, 1985). By late stage 15, DSCl expression is prominent in the frontal ganglionic region (Figs. $1 F, H$; $2 I$ ). Elsewhere in the PNS, some scattered DSCl-expressing cells (Fig. $2 G, I$ ) can be observed that are difficult to identify. A highly reproducible pattern occurs in the abdominal segments where a single $D S C l$-expressing cell is present immediately ventral to every lateral chordotonal organ. Because these cells are anti-HRP negative, their identity as sensory neurons is uncertain.

In the CNS, DSCl expression is detected during germ band retraction as a single cluster per segment along the ventral nerve cord (Fig. $1 D$ ). By stage 15, DSCl is expressed in a regular pattern in some cells located along the longitudinal commissures of the CNS, as revealed by double labeling with anti-HRP (Fig. $2 C, D$ ). In abdominal segments Al-A7, a pair of $D S C$ C - expressing cells lies between the anterior and posterior commissures, very close to the longitudinal commissures and dorsal to the para-expressing neurons. This pattern was not evident in the thoracic segments or in the terminal abdominal segment. Although it is difficult to determine their exact identity, the location of these $D S C 1$-expressing cells suggests they could correspond to longitudinal glia (Klambt and Goodman, 1991).

The results of these in situ hybridization experiments indicate that para and $\mathrm{DSCl}$ are expressed in different subsets of cells in the embryonic CNS and PNS.

\section{Differential expression of para and DSCl continues through the larval stage}

We examined whether the differential expression of para and DSCI continues through later developmental stages. In situ ex- pression patterns of para and $D S C 1$ were studied in third instar larvae using whole-mounts or plastic sections. Expression of para was detected in larval brain, ventral ganglia, eye disc, and leg disc (Fig. 3). In each brain hemisphere, para is expressed in the cellular cortical region and in the developing medullar and laminar regions of the visual system (Fig. $3 A$ ). The inner and outer proliferation centers of the optic lobes, which consist of clustered neuroblasts (White and Kankel, 1978) do not exhibit para expression. In the ventral ganglion, para expression occurs in a segmentally repeated pattern that includes medial, ventrolateral, and dorsolateral neurons (Fig. $3 \mathrm{C}$ ). This staining pattern appears to reflect the continued expression of para in embryonically derived neurons. The vast majority of cells in the ventral ganglion of third instar larvae are produced by larval neuroblasts that undergo mitotic activity throughout larval development (Truman and Bate, 1988). The progeny cells, which will form the adult ventral CNS, accumulate in an immature, arrested state that do not differentiate into mature neurons until metamorphosis. No detectable expression of para is found in these postembryonically derived neuronal precursors before pupal development. Thus, expression of para in the larval CNS is limited to mature neurons that regulate the behavior of the larva.

In leg discs, several para-expressing cells are detected near the larval nerve that connects the disc with the CNS (Fig. 3D). These cells appear to be larval sensory neurons, which are born early during embryogenesis (Jan et al., 1985; Tix et al., 1989). Expression of para is also detected in the developing eye imaginal disc (Fig. $3 E$ ). During the third larval instar, development of ommatidia begins within the morphogenetic furrow as it advances across the disc in a posterior-to-anterior direction (Ready et al., 1976). Randomly arranged undifferentiated cells are located anterior to this furrow. Cellular differentiation and the ordered assembly of cell clusters in each ommatidium begin posterior to the furrow (Ready et al., 1976). Immediately posterior to the furrow, preclusters containing five photoreceptor cells are formed. A second wave of mitosis following the furrow gives rise to three other photoreceptor cells, which are added to the preclusters to form clusters of eight photoreceptors. Posterior to the second mitotic wave, a group of para-expressing cells, corresponding to the developing photoreceptors, can be seen in each ommatidium. However, little or no para expression is detectable in the most posterior part of the eye disc. Thus, para expression appears to diminish as the ommatidial clusters mature further and remains off until late pupal stages (see below).

No para expression is evident in the other imaginal discs, indicating that sensory neurons in these discs may not yet be present. This agrees with the observation that sensory neurons in the wing and haltere discs appear only after the onset of metamorphosis (Tix et al., 1989; Huang et al., 1991).

There is no detectable expression of $D S C I$ in the ventral ganglion or imaginal discs in third instar larvae (data not shown). In the brain, the only detectable expression of $D S C 1$ is in the laminar region of the optic lobes (Fig. $3 B$ ) where it appears to be even more abundantly expressed than para. Although it is difficult to make quantitative comparisons of gene expression on the basis of tissue in situ hybridization, the reproducibility

\footnotetext{
16 embryo hybridized with para probe. Strong expression is seen in the antennomaxillary complex and throughout the entire PNS and CNS. $F$, Stage 16 embryo hybridized with $D S C 1$ probe. Some expression is detected in the CNS and frontal ganglion (arrows) but only faintly elsewhere in the PNS. $G$ and $H$ are ventral views of stage 16 wild-type embryos oriented with anterior to the left. $G$, Hybridization with para probe. $H$, Hybridization with $D S C I$ probe. I, Lateral view of stage 16 para $^{k 2}$ embryo hybridized with para probe. Staining is absent in the mutant embryo.
} 

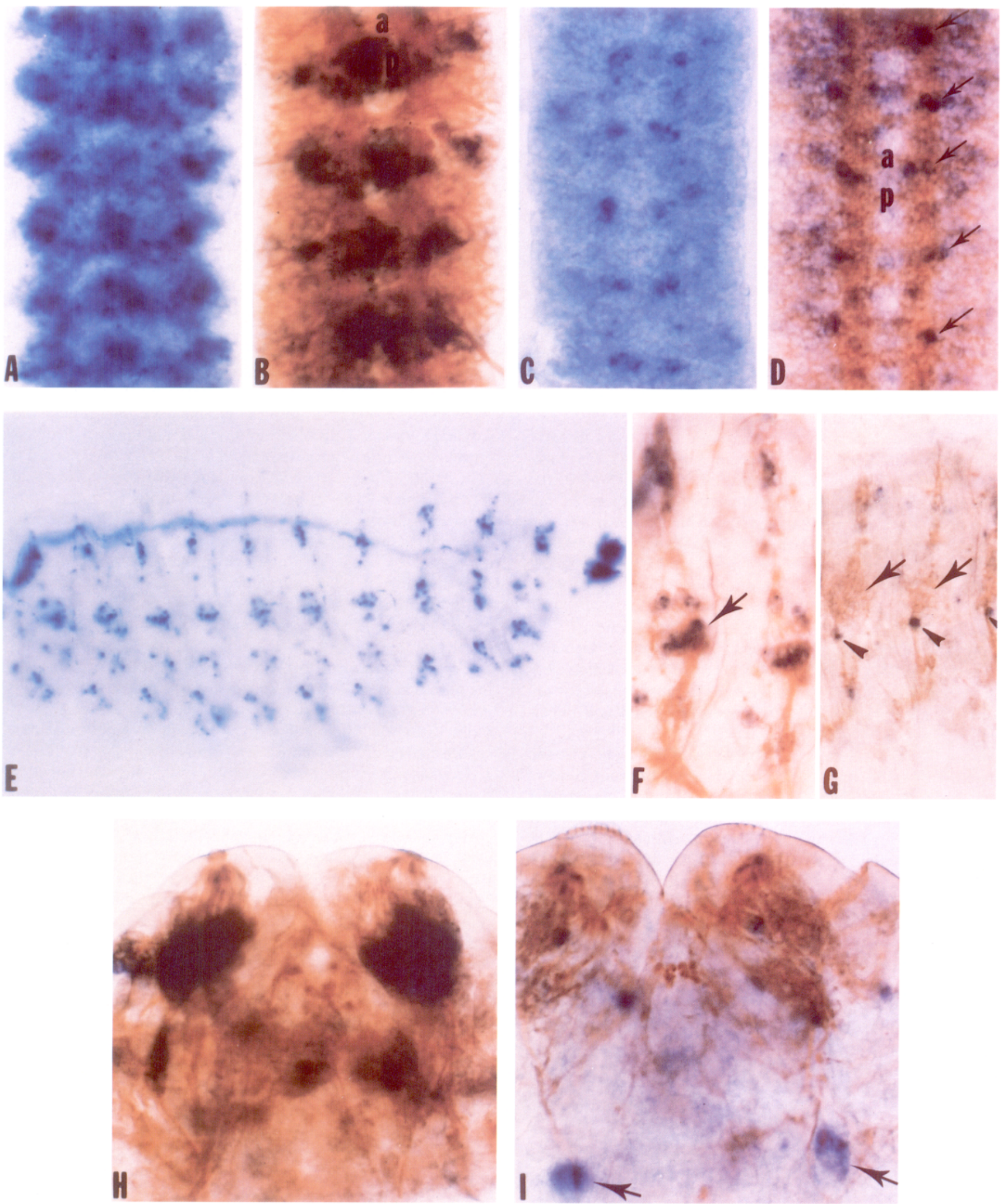

Figure 2. Higher-resolution analysis of para- and DSCl-expressing cells in the embryonic CNS and PNS by in situ hybridization and double labeling with anti-HRP antibodies. $A-D$ show ventral views of expression in ventral ganglion dissected from stage 17 embryos oriented with anterior toward the top. $A$, In situ hybridization with para probe. $B$, Double labeling: in situ hybridization with para probe and immunostaining with antiHRP antibody (brown color) to show location of para-expressing cells relative to axonal tracts. The anti-HRP antibody highlights the longitudinal and anterior $(a)$ and posterior $(p)$ transverse commissures. These axon tracts run between the cell clusters showing strong staining for the para 

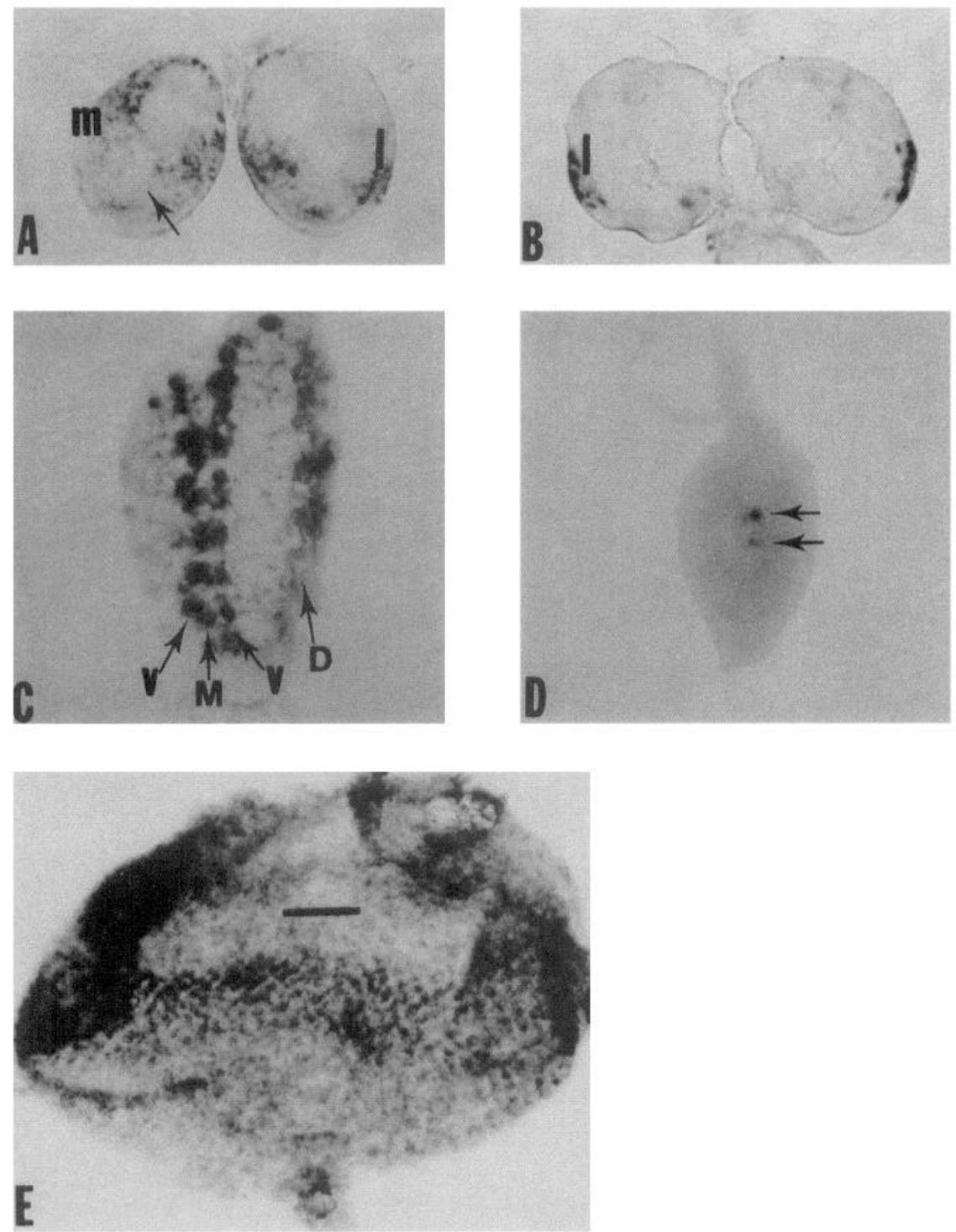

of the intense $D S C l$ staining in the lamina suggests that this is one region where its expression is at higher levels than para. As in embryos, there is little overlap between para and DSC1 expression in the larval nervous system.

\section{Overlapping expression patterns of para and DSC1 in early pupae}

Expression of para and DSC1 was examined in the pupal nervous system beginning at $25 \mathrm{hr}$ after pupariation. At this stage, para transcripts are detected throughout the cellular cortex of the brain, in antennal neurons and also in the optic lobes (Fig.
Figure 3. Comparison of para and DSCl expression in third instar larvae. $A-C$ are horizontal sections through the larval brain and ventral ganglion at about midlevel. Anterior is up. $A$ and $C$, Expression of para in the larval brain hemispheres and the ventral ganglion. Expression is detected in the cortical region and developing laminar $(l)$ and medullar region $(m)$. The inner and outer proliferation centers (arrow) show no staining. In the ventral ganglion, para expression is seen in medial $(M)$, ventrolateral $(V)$, and dorsolateral $(D)$ neurons. $B$, Expression of $D S C 1$ in brain hemispheres. Note prominent expression in the developing laminar region (l). D, para expression (arrows) in the leg disc and $E$, in eye disc whole-mount in situ hybridized. Short line marks the morphogenetic furrow. Posterior is down. Note the faint staining in the very posterior region of eye disc.
$4 A$ ). By this stage, the visual system has developed highly ordered structures in which visual information will undergo serial processing (Kankel et al., 1980). The R1 through R6 photoreceptors terminate in the lamina from which laminar neuropil extends to the medulla. The R7 and R8 photoreceptors synapse directly in the medulla. Neurons in the medulla form synaptic connections in the lobula and lobular plate. Expression of para is detected throughout the cortices of the lamina, medulla, and lobula complex. However, no significant signal is detectable in the retinal region. In young pupae the retina is not differentiated completely and is very thin. Later, about $72 \mathrm{hr}$ after pupariation,

transcript. $C$, In situ hybridization with $D S C 1$ probe. $D$, Double labeling: in situ hybridization with $D S C 1$ probe and immunostaining with antiHRP antibody. DSC1-expressing cells (arrows) are located along the longitudinal commissures close to the anterior transverse commissures. $E-G$ show lateral views of expression in the PNS of stage 17 wild-type embryos oriented with anterior to the left and ventral down. E, In situ hybridization with para probe. $F$, Double labeling: in situ hybridization with para probe and immunostaining with anti-HRP antibody. There is good correspondence between the para-expressing cells and those stained with the anti-HRP antibody. For comparison with DSC1 expression in $G$ below, note expression of para in the chordotonal organ (arrow). $G$. Double labeling: in situ hybridization with $D S C 1$ probe and immunostaining with anti-HRP antibody. Few or none of the cells stained with the anti-HRP antibody have detectable levels of $D S C I$ expression. A DSCI-expressing cell (arrowhead), which is not stained with the antibody, is located ventral to every lateral chordotonal organ (arrows). $H$, Colabeling of paraexpressing cells with anti-HRP antibody in the antennomaxillary complex. Anterior is up. Dorsal focus. I, Expression of DSC1 in the frontal ganglion (arrows). Double labeling: in situ hybridization with $D S C 1$ probe and immunostaining with anti-HRP antibody. 


\section{para}
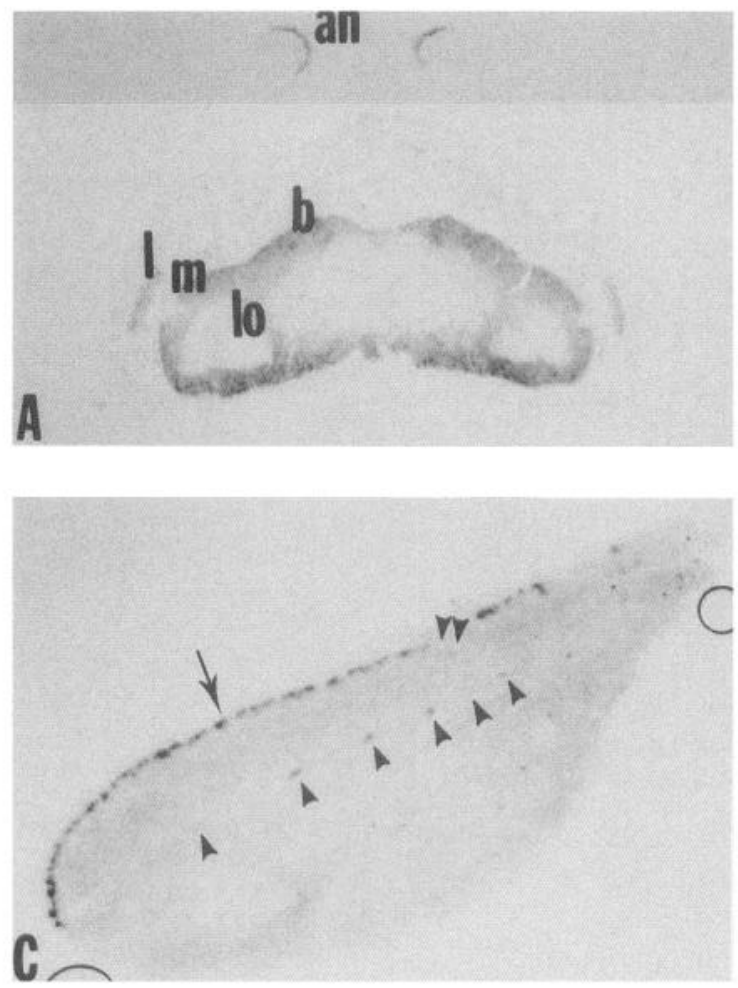

DSC1
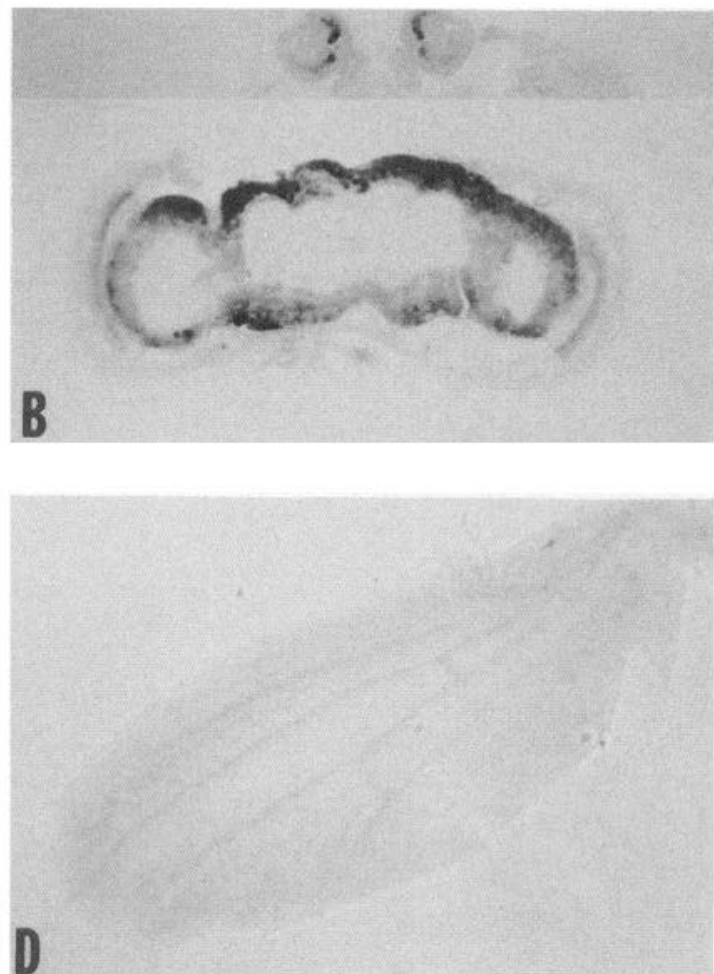

Figure 4. Expression of para and $D S C 1$ in $25 \mathrm{hr}$ pupae. $A$ and $C$ show para expression, and $B$ and $D, D S C 1$ expression. $A$ and $B$ are horizontal sections through the middle of pupal heads. Anterior is up. $A$, In situ hybridization with para probe. Staining appears over the cortical region of brain $(b)$, lamina $(l)$, medulla $(m)$, lobular complex $(l o)$, and also antennal neurons $(a n)$. $B$, In situ hybridization with $D S C 1$ probe. The pattern of DSCl expression appears to overlap with para. $C$ and $D$ show whole-mounts of pupal wings hybridized with para or DSC1 probes. $C$, para probe. Note expression of para in the campaniform sensilla (arrowheads) along the third wing vein as well as in the sensory neurons along the anterior wing margin (arrow). $D, D S C l$ probe. No expression of $D S C l$ is detected in sensory neurons of pupal wings.

when the retina grows rapidly in length and undergoes final differentiation (Cagan and Ready, 1989), expression of para in this region becomes apparent (Fig. $5 \mathrm{~A}$; see also below).

In contrast to the limited expression of $D S C I$ in embryos and larvae, its expression undergoes a substantial change beginning at the pupal stage. By $25 \mathrm{hr}$ after pupariation, $D S C 1$ expression becomes prominent in the CNS and does not differ noticeably from that of para in the brain and optic regions (Fig. $4 B$ ). At this stage, $D S C 1$ is also expressed in antennal neurons in a pattern similar to para.

However, the expression of para and DSC1 is still distinctive in some sensory neurons of the PNS such as in the developing pupal wing (Fig. $4 C, D$ ). A small array of well-defined sensory neurons is present in the developing wing $25 \mathrm{hr}$ after pupariation (Murray et al., 1984). These include sensory neurons along the anterior margin of the wing and a group of eight campaniform sensilla along the third wing vein. All the marginal sensory neurons and all eight campaniform sensilla clearly express para (Fig. $4 C$ ). However, $D S C 1$ expression is not detected in any of these sensory neurons (Fig. $4 D$ ).

\section{Expression of para and DSC1 converges in late pupae and adults}

In $72 \mathrm{hr}$ pupae and adults, para and $D S C 1$ transcripts are both present throughout the brain and thoracic ganglion (Fig. 5). Both transcripts are detected in the cortices of the lamina, medulla, and lobula complex as well as in the retina (Fig. $5 A, B, E, F$ ). Expression of both genes was detected in the retina in layers corresponding to the positions of the cell bodies of photoreceptors R1-R6, R7 and R8 (Fig. 5G,H). The neurons associated with the mechanosensory bristles of the eye also express both para and $D S C 1$. Thus, in contrast with the distinct expression patterns of para and DSC1 observed in earlier developmental stages, in late pupae and adults the expression of these two genes appears to be largely coextensive. Furthermore, from the pupal through adult stages, the intensity of the $D S C l$ signal is reproducibly somewhat greater than that of para, suggesting that DSCl may be expressed at higher levels than para during these stages.

\section{Discussion}

We have examined the spatial and temporal expression of two Drosophila sodium channel genes, para and $D S C 1$, in embryonic through adult stages. The para locus appears to encode the predominant class of sodium channels that is expressed ubiquitously in neurons of the CNS and PNS during all developmental stages. These results are in good agreement with conclusions based on previous genetic and electrophysiological analysis of para mutants. Homozygotes for para null mutations have an unconditionally recessive lethal phenotype and die as first instar larvae (B. Ganetzky, unpublished observations). Temperature-sensitive alleles, such as $\operatorname{para}^{\prime s l}$, cause rapid pa- 
ralysis of both larvae and adults at the restrictive temperature, as a result of a temperature-dependent block in the propagation of action potentials (Suzuki et al., 1971; Siddiqi and Benzer, 1976; Wu and Ganetzky, 1980; Benshalom and Dagan, 1981). Thus, it is likely that most, if not all, neurons require para expression for normal membrane excitability.

Despite the expression of para in most neurons, these neurons may not all express identical para polypeptides. The para transcript has been shown to undergo extensive alternative splicing and at least 18 different coding sequences have been detected in vivo (Loughney et al., 1989; Thackeray and Ganetzky, 1994). Furthermore, the relative frequencies of the various splice forms differ in embryos and adults (Thackeray and Ganetzky, 1994). Thus, temporal as well as spatial differences in splicing of the para transcript may contribute to the distinctive properties of different neurons. Differential distribution in the nervous system of splice variants from the $S h$ potassium channel locus has previously been reported in Drosophila (Schwarz et al., 1990).

Although $\mathrm{DSCl}$ also appears to encode a sodium channel polypeptide (Salkoff et al., 1987), its expression pattern is distinct from that of para. In embryos, only a small number of cells have detectable $D S C 1$ expression. In addition, most of the $D S C 1$-positive cells are not detected by a neural-specific antibody. It may be that the $D S C 1$-expressing cells in the embryonic ventral nerve cord correspond to longitudinal glial cells. The cells in the PNS ventral to the chordotonal organs that express $D S C l$ may also be some type of non-neuronal supporting cell that has so far not been identified. Vertebrate glial cells in the PNS and CNS are known to express voltage-gated ion channels including sodium channels (Gautron et al., 1992; Ritchie, 1992). If the embryonic expression of $D S C 1$ is mostly limited to a few non-neuronal cells, this would explain why cultured neurons from embryos homozygous for a $D S C 1$ deletion expressed sodium currents of normal amplitude (Germeraad et al., 1992).

The failure to detect significant levels of $\mathrm{DSCl}$ expression in embryonic neurons does raise one puzzle. Egg hatch studies have shown that para null embryos survive through the completion of embryogenesis and up to $50 \%$ of these embryos hatch from the egg (Ganetzky, unpublished results). The larvae that emerge are extremely sluggish, fail to molt, and eventually die. Yet, the fact that a significant fraction manage to hatch indicates that para null larvae are able to perform the motor activity required to get out of the egg case. Injection of tctrodotoxin in maturc embryos blocks the peristaltic contractions required for hatching, indicating that propagation of sodium channel-dependent action potentials is necessary to produce these movements (Keshishian et al., 1993). Analysis of null embryos produced by females that are germline mosaics for a para null allele (Ganetzky, unpublished results) and the in situ data reported here eliminate the possibility of maternally contributed para transcripts. Thus, one possible explanation for the ability of para null embryos to hatch is that sodium channels encoded by another gene provide the necessary function. A priori, $D S C 1$ appeared to be the most plausible candidate to fulfill such a role. However, this interpretation now appears unlikely on the basis of the embryonic $D S C l$ expression pattern found here. Instead, we suggest the possible existence of at least one other sodium channel structural gene in Drosophila that is expressed in embryos. It will be of interest to try to identify this postulated gene.

Expression of para in the CNS of embryos and larvae indicates that it is transcribed in neurons but not in neuroblasts or ganglion mother cells. Thus, para does not appear to be expressed in these cells before they begin to become electrically active. Nonetheless, expression of para can be detected in photoreceptors of the developing eye disc. By this stage, the neuronal identity of these cells has already been specified (Venkatesh et al., 1985), although it is unclear whether they have yet become electrically active. Transcription of para in these cells wanes as the ommatidial cluster matures such that very little or no para expression is detected in the most posterior region of the eye disc. Furthermore, no significant expression of para could be detected in photoreceptors of $25 \mathrm{hr}$ pupae. Thus, it appears that there is a very short period of para expression during the early differentiation of photoreceptor cells. Subsequently, expression of para is reduced or absent in these cells until late pupal stages when strong expression resumes. Although both para and DSC1 are expressed in photoreceptors of late pupae, only para is expressed in the developing eye disc. To examine the potential significance of para expression in the developing eye disc, we generated mosaic patches homozygous for a para null mutation. However, this elimination of para expression in the eye disc did not cause any gross abnormalities in development of the retina or in any of the downstream optic ganglia. Whether there might be more subtle defects in these mosaic patches, such as perturbation of axonal pathfinding, has not been examined.

Aside from the question of early para expression in the eye disc, expression of both para and DSCI transcripts in fully mature photoreceptors was unexpected. In Diptera, as in other insects, transmission of the receptor signal down the retinula cell axon to the synaptic ending is thought to depend on passive spread of the receptor potential (Pak and Grabowki, 1978). Intracellular recordings from dipteran photoreceptors have never indicated the occurrence of action potentials (Pak and Grabowski, 1978). Furthermore, the presence of voltage-activated sodium channels was not evident in whole-cell patch-clamp recordings of dissociated Drosophila photoreceptors (Hardie, 1991). However, voltage-activated sodium channels have been found in photoreceptors of drone honeybees where they are involved in the amplification of small signals (Vallet et al., 1992). Because drone photoreceptors have evolved for the effective performance a specific task-detection of spots in the sky that might be queen bees-it is not clear whether a comparable amplification mechanism occurs, or is required, in Drosophila. Thus, the role of sodium channels in the development and function of Drosophila photoreceptors is an interesting question for further study.

In pupae and adults, expression of para and $D S C I$ remains different in the PNS but overlaps almost completely in the CNS. An important neuronal function for $D S C l$ is suggested by its strong and widespread expression in the pupal and adult CNS. Why do most, if not all, CNS neurons express two sodium channel genes? Because the polypeptides encoded by para and $D S C 1$ are quite distinct and have probably been diverging from each other for at least 600 million years, they may have different properties enabling them to subserve different physiological functions - a conclusion supported by the results of gene dosage studies (Stern et al., 1990). For example, a single neuron could express two types of sodium channels, one targeted for axons and one for cell bodies or terminals. Distribution to different functional domains has been demonstrated for two members of the $S h$ family of potassium channels in hippocampal neurons (Sheng et al., 1992). Determination of the subcellular distribution of para and $D S C l$ polypeptides with type-specific antibodies would be informative in this regard. On the other hand, 


\section{para}
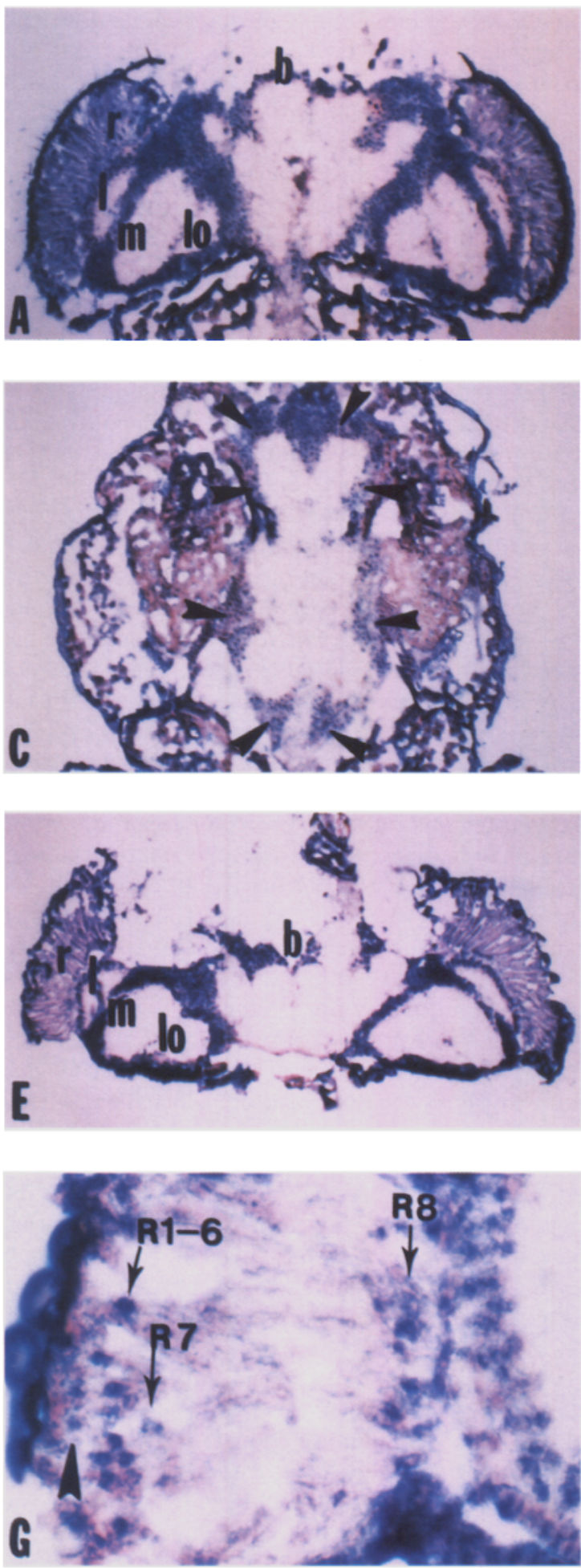

DSC1
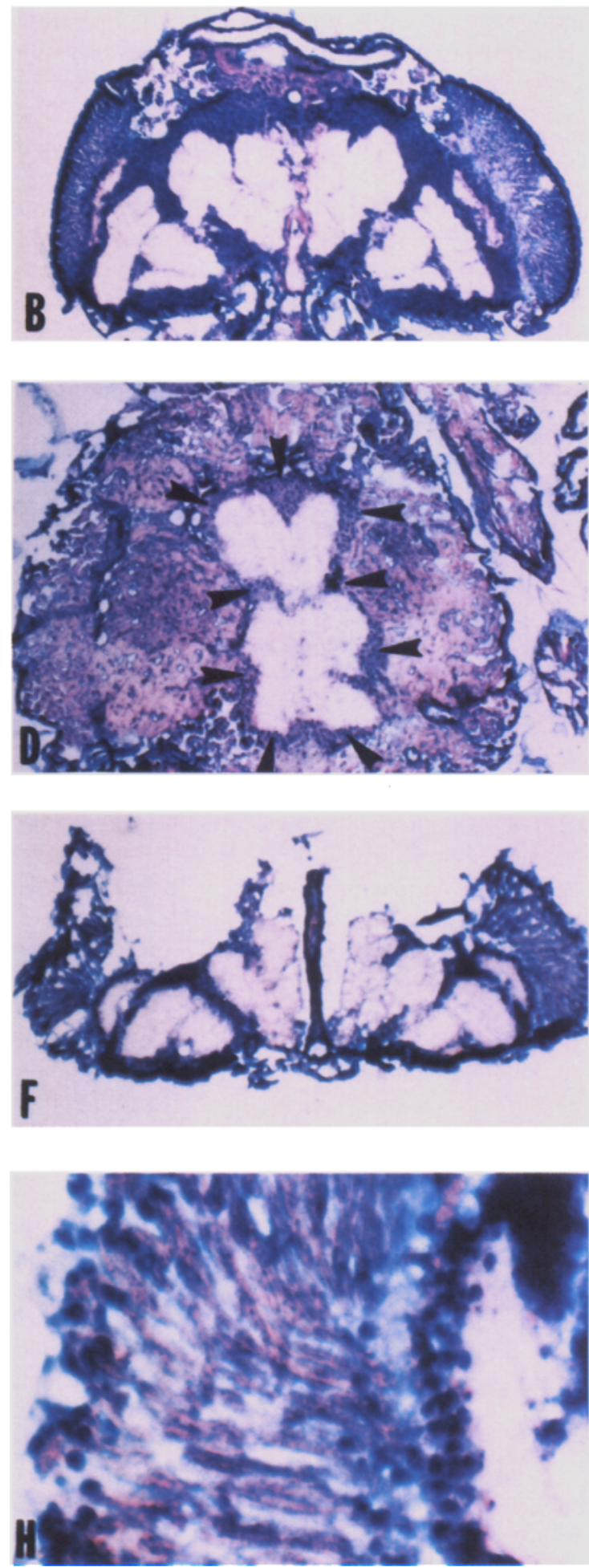

Figure 5. Expression of para and DSC1 in $72 \mathrm{hr}$ pupae and in adult heads. Left panels $(A, C$, E, and $G)$ show para expression, and right panels $(B, D, F$, and $H), D S C 1$ expression. $A$ and $B$ are horizontal sections through the middle of pupal heads. $C$ and $D$ are horizontal sections through the middle of thoraces. $E-H$ are horizontal sections through the middle of adult heads about $4 \mathrm{~d}$ after eclosion. Anterior is up. $A$, Hybridization with para probe. $B$, Hybridization with $D S C 1$ probe. Expression of both para and $D S C l$ can be detected in cellular cortices of brain $(b)$, retina $(r)$, lamina $(l)$, medulla $(m)$, and the lobular complex $(l o)$. Note that expression in the retina is detected in $72 \mathrm{hr}$ pupae, although it was absent earlier in $25 \mathrm{hr}$ pupae. $C$, Hybridization with para probe. $D$, Hybridization with $D S C 1$ probe. Both para and $D S C 1$ are expressed in the cellular cortex of thoracic ganglion (arrowheads) but neither gene has detectable expression in cells outside the nervous system. $E$, Hybridization with para probe. $F$, Hybridization with $D S C 1$ probe. For both para and $D S C 1$ the staining pattern in the cellular cortices of brain $(b)$, retina $(r)$, lamina $(l)$, medulla $(m)$, and lobular complex $(l o)$ are all the same as in late pupal stages. $G$ and $H$ show a higher magnification of the retinal region. $G$, Hybridization with para probe. $H$, Hybridization with $D S C 1$ probe. The photoreceptors, R8 and R7 as well as R1-R6 located in a layer just distal to R7 are all stained. The sensory neurons of the eye bristle (arrowhead) close to the photoreceptors R1-R6 seems to be stained. 
some functional overlap between para and $D S C 1$ could help explain the persistence of action potentials in neurons of the flight motor pathway in para $^{\text {ts }}$ adults at restrictive temperatures (Elkins and Ganetzky, 1990). Isolation of $D S C 1$ mutations would be very useful in addressing its functional role in vivo.

\section{References}

Amichot M, Castella C, Berge JB, Pauron D (1993) Transcription analysis of the para gene by in situ hybridization and immunological characterization of its expression product in wild-type and mutant strains of Drosophila. Insect Biochem Mol Biol 23:381-390.

Benshalom G, Dagan D (1981) Electrophysiological analysis of the temperaturc-scnsitive paralytic Drosophila mutant, para ${ }^{\prime *}$. J Comp Physiol 144:409-417.

Cagan RL, Ready DF (1989) The emergence of order in the Drosophila pupal retina. Dev Biol 136:346-362.

Campos-Ortega JA, Hartenstein V (1985) Stages of Drosophila embryogenesis. In: The embryonic development of Drosophila melanogaster, pp 9-84. New York: Springer.

Campos-Ortega JA, Jan YN (1991) Genetic and molecular bases of neurogenesis in Drosophila melanogaster. Annu Rev Neurosci 14: $399-420$.

Elkins T, Ganetzky B (1990) Conduction in the giant nerve fiber pathway in temperature-sensitive paralytic mutants of Drosophila. $\mathrm{J}$ Neurogenet 6:207-219.

Gautron S, Santos GD, Pinto-Henrique D, Koulakoff A, Gros F, Berwald-Netter Y (1992) The glial voltage-gated sodium channcl: celland tissue-specific mRNA expression. Proc Natl Acad Sci USA 89: $7272-7276$.

Germeraad S, O'Dowd DK, Aldrich RW (1992) Functional assay of a putative Drosophila sodium channel gene in homozygous deficiency neurons. J Neurogenet 8:1-16.

Hafen E, Levine M (1986) The localization of RNAs in Drosophila tissue sections by in situ hybridization. In: Drosophila, a practical approach (Roberts DB, ed), pp 139-157. Oxford: IRL.

Hardie RC (1991) Voltage-sensitive potassium channels in Drosophila photoreceptors. J Neurosci 11:3079-3095.

Hartenstein V (1988) Development of Drosophila larval organs: spatiotemporal pattern of sensory neurons, peripheral axonal pathways and sensilla differentiation. Development 102:869-886.

Hille B (1992) Ion channels of excitable membranes, $2 \mathrm{~d}$ cd. Sunderland, MA: Sinauer.

Huang F, Dambly-Chaudiere C, Ghysen A (1991) The emergence of sense organs in the wing disc of Drosophila. Development 111:10871095.

Jan LY, Jan YN (1982) Antibodies to horseradish peroxidase as specific neuronal markers in Drosophila and in grasshopper embryos. Proc Natl Acad Sci USA 72:2700-2704.

Jan YN, Ghysen A, Christoph I, Barbel S, Jan LY (1985) Formation of neuronal pathways in the imaginal discs of Drosophila melanogaster. J Neurosci 5:2453-2464.

Kallen RG, Sheng Z-H, Yang J, Chen L, Rogart RB, Barchi RL (1990) Primary structure and expression of a sodium channel characteristic of denervated and immature rat skeletal muscle. Neuron 4:233-242.

Kankel DR, Ferrus A, Garen SH, Harte PJ, Lewis PE (1980) The structure and development of the nervous system. In: The genetics and biology of Drosophila, Vol 2d (Ashburner M, Wright TRF, eds), pp 295-368. New York: Acadernic.

Kayano T, Noda M, Flockerzi V, Takahashi H, Numa S (1988) Primary structure of rat brain sodium channel III deduced from the cDNA sequence. FEBS Lett 228:187-194.

Keshishian H, Chiba A, Chang TN, Halfon MS, Harkins EW, Jarecki J, Wang L, Anderson M, Cash S, Halpern ME, Johansen J (1993) Cellular mechanisms governing synaptic development in Drosophila melanogaster. J Neurobiol 24:757-787.

Klambt C, Goodman CS (1991) The diversity and pattern of glia during axon pathway formation in the Drosophila embryo. Glia 4:205213.

Loughney K, Kreber R, Ganetzky B (1989) Molecular analysis of the para locus a sodium channel gene in Drosophila. Cell 58:1143-1154.

Mandel G (1992) Tissue-specific expression of the voltage sensitive sodium channel. J Membr Biol 125:193-205.

Masucci JD, Miltenberger RJ, Hoffmann FM (1990) Pattern-specific expression of the Drosophila decapentaplegic gene in imaginal disks is regulated by 3' cis-regulatory elements. Genes Dev 4:2011-2023.

Murray MA, Schubiger M, Palka J (1984) Neuron differentiation and axon growth in the developing wing of Drosophila melanogaster. Dev Biol 104:259-273.

Noda M, Ikeda T, Kayano T, Suzuki H, Takeshima H, Kurasaki M, Takahashi H, Numa S (1986) Existence of distinct sodium channel messenger RNAs in rat brain. Nature 320:188-192.

Pak WL, Grabowski SR (1978) Physiology of the visual and flight systems. In: The genetics and biology of Drosophila, Vol 2a (Ashburner M, Wright TRF, eds), pp 553-604. New York: Academic.

Ramaswami M, Tanouye M (1989) Two sodium channel genes in Drosophila: implications for channel diversity. Proc Natl Acad Sci USA 86:2079-2082.

Ready DF, Hanson TE, Benzer S (1976) Development of the Drosophila retina, a neurocrystalline lattice. Dev Biol 53:217-240.

Reuter R, Scott MP (1990) Expression and function of the homoetic genes Antennapedia and Sex combs reduced in the embryonic midgut of Drosophila. Development 109:289-303.

Ritchie JM (1992) Voltage-gated ion channels in Schwann cells and glia. Trends Neurosci 15:345-350.

Rogart RB, Cribbs LL, Muglia LK, Kephart DD, Kaiser MW (1989) Molecular cloning of a putative tetrodotoxin-resistant rat heart $\mathrm{Na}^{+}$ channel isoform. Proc Natl Acad Sci USA 86:8170-8174

Salkoff L, Butler A, Wei A, Scavarda N, Giffen K, Ifune C, Goodman R, Mandel G (1987) Genomic organization and deduced amino acid sequence of a putative sodium channel gene in Drosophila. Science 237:744-749.

Schwarz TL, Papazian DM, Carretto RC, Jan YN, Jan LY (1990) Immunological characterization of $\mathrm{K}^{+}$channel components from the Shaker locus and differential distribution of splicing variants in Drosophila. Neuron 2:119-127.

Sheng M, Tsaur M, Jan YN, Jan LY (1992) Subcellular segregation of two A-type $\mathrm{K}^{+}$channel proteins in rat central neurons. Neuron $9: 271-284$

Siddiqi O, Benzer S (1976) Neurophysiological defects in temperaturesensitive mutants of Drosophila melanogaster. Proc Natl Acad Sci USA 73:3253-3257.

Stern M, Kreber R, Ganetzky B (1990) Dosage effects of a Drosophila sodium channel gene on behavior and axonal excitability. Genetics 124:133-143.

Suzuki DT, Grigliatti T, Williamson R (1971) Temperature-sensitive mutations in Drosophila melanogaster, VII. A mutation ( para $^{t s}$ ) causing reversiblc adult paralysis. Proc Natl Acad Sci USA 68:890-893.

Tautz D, Pfeifle C (1989) A non-radioactive in situ hybridization method for the localization of specific RNAs in Drosophila embryos reveals translational control of the segmentation gene hunchback. Chromosoma 98:81-85.

Thackeray JR, Ganetzky B (1994) Developmentally regulated alternative splicing generates a complex array of Drosophila para sodium channel isoforms. J Neurosci 14:2569-2578.

Tix S, Bate M, Technau GM (1989) Pre-existing neuronal pathways in the leg imaginal discs of Drosophila. Development 107:855-862.

Trimmer JS, Cooperman SS, Tomiko SA, Zhou J, Crean SM, Boyle MB, Kallen RG, Sheng Z, Barchi RL, Sigworth FJ, Goodman RH, Agnew WS, Mandel G (1989) Primary structure and functional expression of a mammalian skeletal muscle sodium channel. Neuron 3:33-49.

Truman J, Bate M (1988) Spatial and temporal patterns of neurogenesis in the central nervous system of Drosophila melanogaster. Dev Biol 125:145-157.

Tseng-Crank J, Pollock JA, Hayashi I, Tanouye MA (1991) Expression of ion channel genes in Drosophila. J Neurogenet 7:229-239.

Vallet AM, Coles JA, Eilbeck JC, Scott AC (1992) Membrane conductances involved in amplification of small signals by sodium channels in photoreceptors of drone honey bee. J Physiol (Lond) 456:303324

Venkatesh TR, Zipursky SL, Benzer S (1985) Molecular analysis of the development of the compound eye in Drosophila. Trends Neurosci $8: 251-257$.

White K, Kankel DR (1978) Patterns of cell division and cell movement in the formation of the imaginal nervous system in Drosophila melanogaster. Dev Biol 65:296-321.

Wu C-F, Ganetzky B (1980) Genetic alteration of nerve membrane excitability in temperature-sensitive paralytic mutants of Drosophila melanogaster. Nature 286:814-816. 